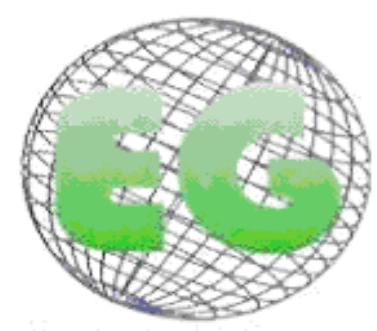

ISSN 1695-6141

$\mathrm{N}^{\circ} 24$
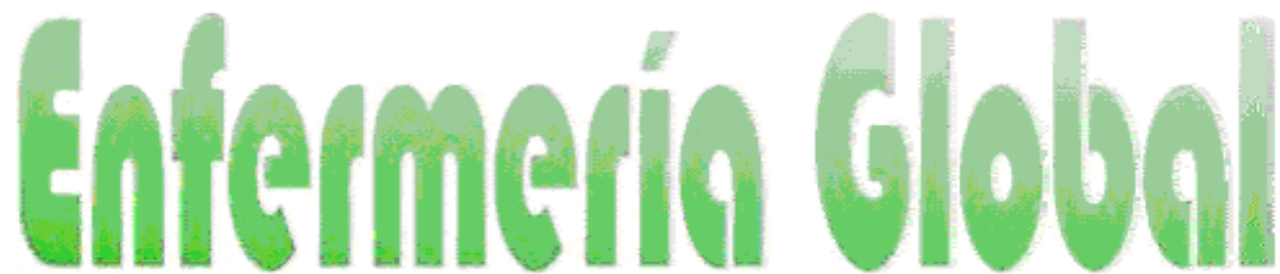

Revista electrónica trimestral de Enfermería

Octubre 2011

www.um.es/egloball

\title{
Una alternativa poco habitual: la vía intraosea
}

An unusual alternative: the intraosseous route

\author{
*Míguez Burgos, A. **Muñoz Simarro, D. ***Tello Pérez, S. \\ "DUE. FREMAP Ciudad Jardín. ** DUE. D. C. C. U. Móviles Distrito. ****Lcda. en Derecho. Sevilla. \\ Palabras clave: Vía intraosea; Acceso vascular; Médula ósea; Técnica inserción; Guía de recomendaciones. \\ Keywords: Intraosseous route: vascular access; bone marrow; insertion technique; recommendations guide
}

\section{RESUMEN}

En el presente artículo, tras una intensa revisión bibliográfica, intentaremos implantar una guía de recomendaciones a la hora de utilizar esta vía, para ello expondremos las principales indicaciones y contraindicaciones de la citada vía, así como las técnicas de inserción y principales regiones anatómicas afectas.

Debemos tener en cuenta que se trata de un acceso vascular de urgencia para la infusión de fármacos y líquidos, por lo que vamos a delimitar sus indicaciones concretas y a quien corresponde su aplicación, así como la descripción de la técnica en todos sus pasos.

La base teórica del uso de esta vía está basada en que la cavidad medular de huesos largos está ocupada por una rica red de capilares sinusoides que drenan al gran seno venoso central y que permite pasar dichos fármacos y líquidos a la circulación general con una rapidez similar a como lo harían por cualquier otra vena periférica.

Se trata de una vía de elección poco conocida por el personal sanitario, pero de gran utilidad en pacientes con difícil acceso venoso por colapso circulatorio de origen patológico o traumático, por lo que tras la elaboración de este artículo pretendemos que a todos nos sea un poco más fácil su uso.

\section{ABSTRACT}

After an intensive literature review, we seek here to implement a recommendation guide when using this route. There is a discussion of the indications and contraindications of the route and insertion techniques and of the key anatomical regions affected.

We should note that this is an emergency vascular access for infusion of drugs and fluids, so we will define the specific indications and who is responsible for its implementation and offer a description of the technique in all its steps. 
The theoretical basis of using this approach is based on the fact that the long bone marrow cavity is occupied by a rich network of sinusoids which drain the large central venous sinus and allowed these drugs and fluids to pass into the general circulation with a speed similar to that at which they would pass to any other peripheral vein.

It is a little-known route of choice by the health staff, but very useful in patients with difficult venous access due to circulatory collapse of pathological or traumatic origin. The article therefore seeks to facilitate its use for all concerned.

\section{INTRODUCCIÓN - JUSTIFICACIÓN: ANTECEDENTES HISTÓRICOS}

Empezaremos recordando los principales aspectos fisiológicos y anatómicos que tratamos en este tema. La cavidad medular de huesos largos se compone de una rica red de capilares sinusoides que drenan al gran seno venoso central y que permite pasar dichos fármacos y líquidos a la circulación general con una rapidez similar a como lo harían por cualquier otra vena periférica; el inicio de acción es también similar a la administración venosa, aunque debemos tener en cuenta que deben ser inyectados a presión para superar la resistencia de las venas emisarias que conectan la cavidad intramedular con la circulación general a través de los vasos microscópicos de la cortical ósea.

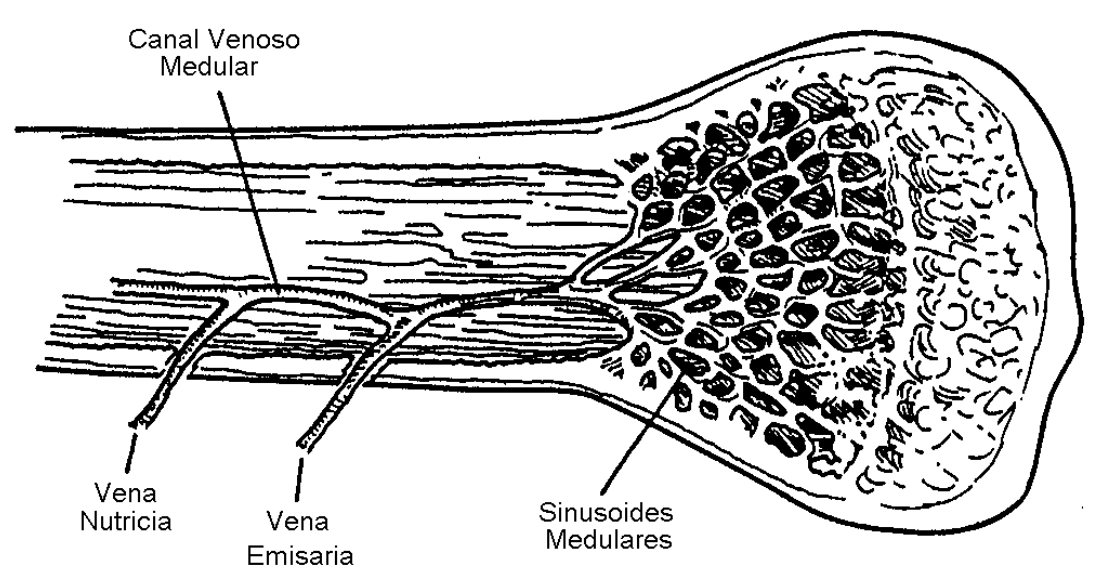

Cuando el profesional sanitario se enfrenta a una situación de emergencia, el establecer un adecuado acceso vascular es tan importante como los otros principios básicos de atención del paciente crítico. Conjuntamente con el resto de medidas de soporte vital avanzado, el tener disponible una vía o acceso para la administración de medicamentos, líquidos o hemoderivados es prioritario para obtener resultados óptimos ante estas situaciones difíciles. ${ }^{(1,2,3,4)}$

Existen situaciones de emergencia donde la obtención de accesos vasculares puede ser difícil, tardío y frustrante: profesionales sanitarios no familiarizados con las técnicas y que no utilizan estos procedimientos con frecuencia, por lo general, fallan o tardan más tiempo en realizarlos, o en determinados casos donde es imposible canalizar los accesos vasculares habituales por diversos motivos, en caso de niños, sumado a que la ansiedad y la urgencia hacen técnicamente más difícil la colocación de un acceso venoso en pacientes muy graves. ${ }^{(4)}$ 
Se trata pues de una vía de elección poco conocida por el personal sanitario, pero de gran utilidad en pacientes con difícil acceso venoso por colapso circulatorio de origen patológico o traumático. Recordando siempre que es de carácter temporal y que debe ser retirada en cuanto el paciente se haya estabilizado y/o hayamos conseguido establecer otros accesos venosos de origen central o periféricos.

\section{Antecedentes historicos}

- 1922: Se describe la anatomía de la médula ósea y su importancia en la perfusión de líquidos en animales de laboratorio.

- 1934: Se usa por primera vez en humanos para tratar una anemia perniciosa

- 1940: Se empieza a utilizar para trasfundir sangre en el tratamiento de la granulopenia

- 1942: Se demuestra la rápida absorción utilizando esta vía y la equivalencia a la perfusión intravenosa

- 1950-1960: Se comienza a reemplazar de forma gradual por el uso de catéteres intravenosos. La aparición de las cánulas venosas de plástico y polifluoroeno facilitó las perfusiones intravenosas prolongadas y las nuevas técnicas de acceso intratecal, intracardiaco, intraperitoneal y sublingual se volvieron populares haciendo disminuir el interés por la vía intraosea

- 1977: Vuelve a aparecer el interés por ésta vía en trabajos con venografía intraosea para la administración de líquidos y medicamentos que no se habían infundido por esta vía como Ringer lactato, solución salina, glucosa, dexametasona, atropina, lidocaína, heparina y diazoxido.

- 1984: Rosetti y sus colaboradores publicaron un estudio retrospectivo de 3 años sobre vías de acceso venosas en niños en reanimación cardiopulmonar. $(7,10)$

- 1985: Este mismo equipo realizó un estudio sobre la utilización de la vía intraosea que demostró sólo un $2,1 \%$ de fallas y $0,6 \%$ de casos de osteomielitis, cifra baja si se tiene en cuenta que esta vía de acceso fue utilizada en muchos casos por tiempo prolongado. ${ }^{(11)}$

Estudios posteriores han confirmado la seguridad y eficacia de esta técnica, con gran velocidad de absorción, la médula permite una perfusión rápida y completa de líquidos y medicamentos, comparable al que se obtiene con una cánula intravenosa de igual calibre. Por lo tanto la médula ósea puede considerarse como una vena rígida que no se colapsa.

\section{METODOLOGÍA}

Revisión Bibliográfica y análisis sobre el tema. Propuesta de actividades.

\section{OBJETIVOS}

Nuestros principales objetivos van a ser los siguientes:

1.- El primer gran objetivo va a ser que el profesional sanitario que se enfrenten a situaciones de emergencia tenga presente la vía intraosea como otra alternativa más de acceso vascular para el suministro de medicación y soluciones.

2.- Conocimiento de todos los aspectos de este acceso tanto en sus indicaciones, contraindicaciones, técnicas de inserción, etc. Si se consigue este objetivo será más fácil de cumplir el primero y tercero ya que con un conocimiento pleno del acceso será más fácil la adquisición de un dominio de la técnica y del acceso y con ello no lo veremos como una alternativa utópica o especial sino cómo otra alternativa más. 
3.- Pleno dominio de la técnica y establecimiento de un protocolo o guía de recomendaciones a la hora de utilizar esta vía.

4.- Establecer sesiones de formación entre el personal sanitario para familiarizar con esta técnica a todo el personal que pueda tener que utilizarla.

\section{RESULTADOS}

\section{Principales indicaciones}

Desde el año 2000 tanto la Asociación Americana del Corazón (AHA) como el Consejo Europeo de Resucitación (ERC) incluyen en sus recomendaciones el uso de esta vía para los casos de parada cardiorrespiratoria, shock, convulsiones intratables, y otros tipos de emergencias. $(1,2,3,4,9,12,13,14)$. Además recomiendan su uso en todos los grupos de edad y no sólo en pediatría como sucedía en sus anteriores recomendaciones y se pueden

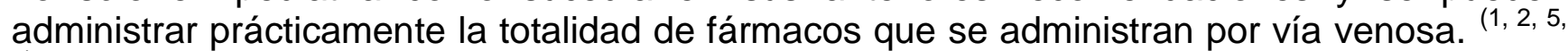

6). Enumeraremos las principales indicaciones.

- Casos en los que no hay posibilidad de obtener acceso venoso por colapso circulatorio, bien sea de origen médico o traumático.

- En pacientes muy graves y tras 90 segundos o 2-3 intentos intentando obtener vía venosa.

- Grandes quemados

- Pacientes en estado de shock que precisen RCP

- Casos que presenten edemas severos

- Recién nacidos en asistolia

- Vía de elección segura en niños de 0 a 6 años, que puede ser recomendable hasta niños de 12 años.

- Poli traumatizados graves

- Hipovolemia

Como nombrábamos anteriormente hay que tener en cuenta que se trata de una medida de carácter temporal, que va encaminada a la reanimación del paciente hasta conseguir una canalización por acceso venoso. No se debe utilizar en huesos fracturados para aumentar volumen circulante aunque sí para administrar analgesia.

Puede administrarse por esta vía cualquier tipo de sueros, tanto cristaloides como coloides, así como sangre y fármacos. En caso de la administración de estos últimos se aconseja administrar un bolo posterior de por lo menos $5 \mathrm{ml}$ de solución salina para asegurar la llegada a la circulación sistémica.

\section{Principales contraindicaciones y complicaciones}

-Fractura en ese mismo hueso

-Celulitis u osteomielitis en el sitio de aplicación

-Osteopetrosis u Osteogénesis imperfecta

-Cuando sea necesaria la administración de bicarbonato sódico y bretilio

-Huesos grandes de las extremidades inferiores en los pacientes con traumatismo abdominal grave.

-Cuando se realizan punciones corticales múltiples se reduce la eficacia de la técnica; por eso, es aconsejable que ante un fallo en la punción se deje la aguja con el estilete en ese mismo lugar, intentando colocar otra en un lugar próximo a ésta. De todas formas se 
recomienda que la segunda punción y las siguientes se produzcan en otra extremidad o en una región del hueso más proximal

\section{Principales complicaciones que pueden surgir}

Los estudios realizados en animales han demostrado que los efectos a largo plazo sobre la médula ósea y sobre el crecimiento óseo son mínimos. ${ }^{(8)}$

El índice de complicaciones de esta técnica invasiva es muy bajo. La más frecuente es la extravasación de líquidos, de medicamentos o de ambos como resultado de una mala colocación de la aguja.

Otras complicaciones son: la celulitis localizada con o sin osteomielitis (en menos de un 1\%, sobre todo en casos de dejar esta vía periodos prolongados), abscesos subcutáneos, síndrome compartimental, fracturas, la lesión al cartílago de crecimiento, sepsis y rara vez la muerte secundaria a la perforación del esternón seguida de mediastinitis, hidrotórax o perforación de los grandes vasos

\section{Materiales y sitios anatómicos de inserción}

Los materiales que vamos a necesitar son los siguientes:

- Agujas de punción intraósea que serán por orden de preferencia:

- Agujas intraóseas especiales con estilete, bisel corto y multiperforadas 15G$18 \mathrm{G}$.

- Trocar para punción de médula ósea 13G-16G.

- Agujas de punción lumbar 18G-20G.

- Agujas hipodérmicas 18G-20G.

- Agujas epicraneales 16G-18G.
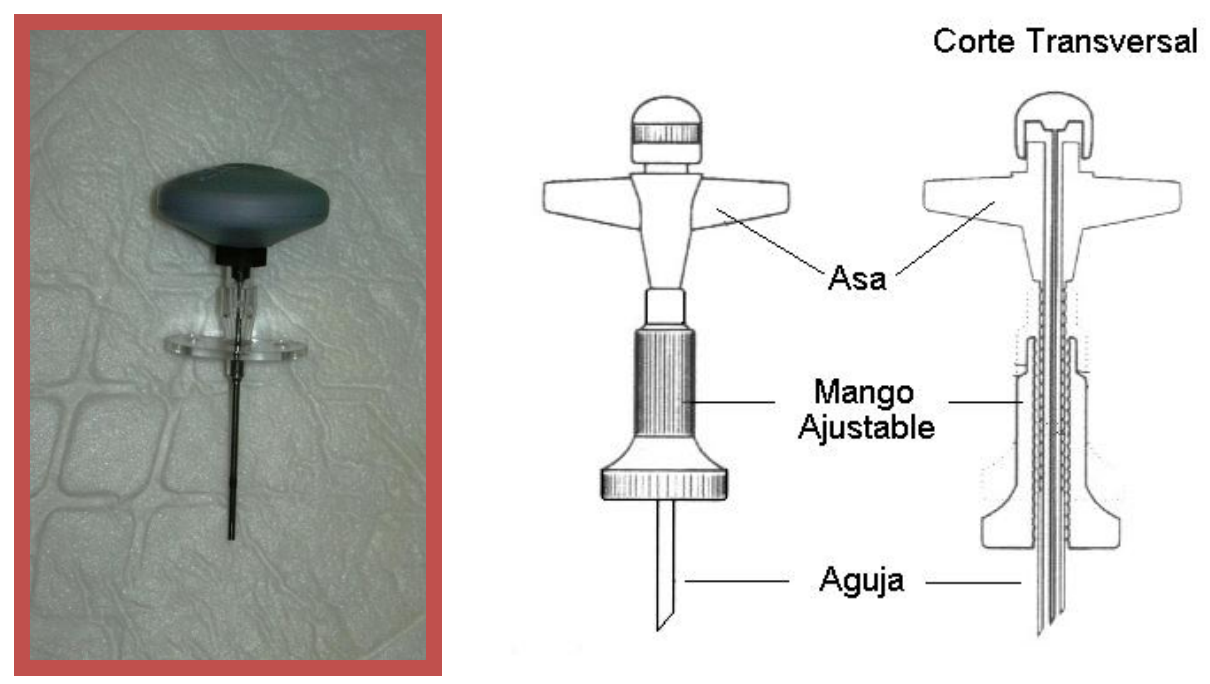


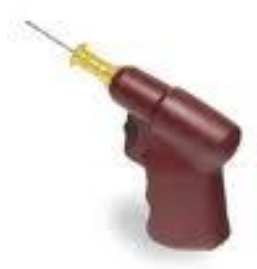

- Existen además dispositivos automáticos de inserción de agujas intraóseas-BIG (Bone Inyection Gun), que según los autores ofrece un acceso rápido y seguro al sistema vascular.
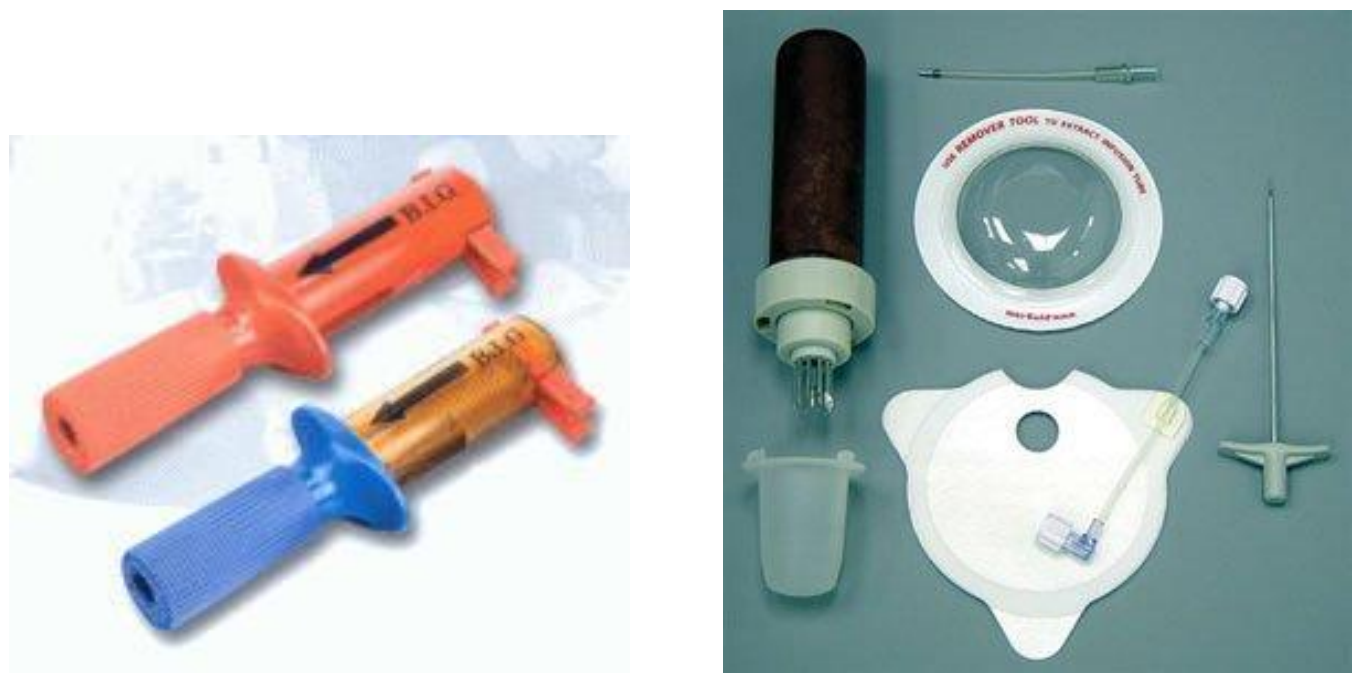

- Jeringas de 5, 10, $20 \mathrm{ml}$ y agujas convencionales.

- Anestésico local.

- Solución antiséptica.

- Guantes estériles.

- Suero fisiológico.

- Sistema de perfusión.

- Gasas y vendas

- Pinza Kocher.

- Esparadrapo.

- Paños estériles

- Llave de tres pasos con alargadera (para no movilizar la aguja sobre el hueso).

Los sitios de inserción son los siguientes.

Se han descrito diferentes sitios para la colocación de la aguja intraosea. En los niños y a medida que se va produciendo el crecimiento, la médula ósea roja vascular cambia a médula 
amarilla menos mascularizada, siendo infiltrada por células grasas. Por lo tanto, muchos lugares que son propicios para ésta técnica en niños desaparecen en el adulto ${ }^{(10)}$. A continuación vamos a diferenciar:

a) En niños hasta 6 años es preferente su aplicación en Tibia proximal, exactamente en la superficie plana antero-interna de la tibia, aproximadamente $1-3 \mathrm{~cm}$ por debajo de la tuberosidad tibial, ya que la cavidad medular en esa localización es grande y la posibilidad de lesión de tejidos adyacentes es mínima $(1,2,8)$. Otras alternativas pueden ser la cara posterior de la metáfisis del radio, cara anterior de la cabeza humeral, cóndilo humeral, fémur distal, esternón y crestas ilíacas.

b) En mayores de 6 años y adultos, se puede insertar además de la tibia proximal, en la distal (1 ó $2 \mathrm{~cm}$ por encima del maléolo interno), radio y cúbito distal, esternón y espina iliaca antero-superior $(1,2)$. Para el profesional resulta más asequible la punción en tibia proximal o distal ya que la piel se encuentra adherida al periostio y sin presencia de grasa intermedia. Este dato es muy a tener en cuenta sobre todo en pacientes obesos.

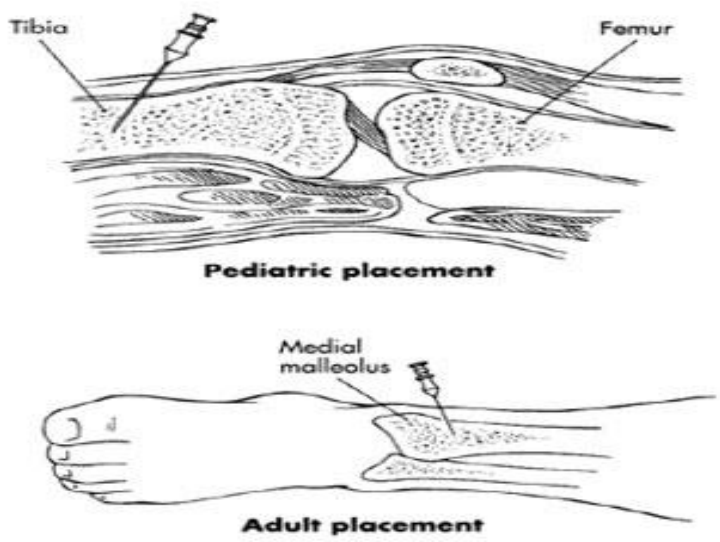

Técnica de inserción ${ }^{(14)}$

1.- Localización del punto de inserción: Si el paciente está consciente habrá que anestesiar previamente la zona. Y Apoyo de la pierna sobre una superficie firme.

2.- Lavado de manos

3.- Limpieza de la piel con solución antiséptica

4.- Colocación de guantes estériles

5.- Sujetar la rodilla por fuera y arriba del sitio de inserción con la mano no dominante.

6.- Agarrar firmemente el catéter en la palma de la mano, apoyando el dedo índice a $1 \mathrm{~cm}$ de la punta de la aguja para evitar profundizar mucho. 7.- Insertar la aguja perpendicularmente al hueso o con un ángulo de $60^{\circ}$ a 75 pero con la punta de la aguja en dirección opuesta a donde se encuentran los cartílagos de crecimiento.

8.- Avanzar la aguja a través de la cortical ósea con un movimiento rotatorio suave pero firme. Se nota una resistencia moderada a la penetración que disminuye repentinamente al atravesar el córtex del hueso (indica que estamos en la cavidad de la médula ósea), a la vez se nota un tacto en el 
catéter como de crujido. En los lactantes, la distancia entre la piel y la trabécula ósea no es mayor de $1 \mathrm{~cm}$.

9.- Desenroscar el extremo posterior de la aguja y retirar el estilete

10.- Aspirar con la jeringa de $10 \mathrm{ml}$ y comprobar la extracción de médula ósea. Este material no debe desecharse ya que es útil para análisis de

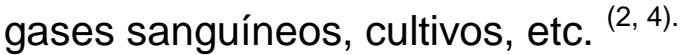

11.- Fijación del catéter al miembro mediante un paquete de gasas y esparadrapo de tela e inmovilización del miembro con una férula y una venda.

12.- Inyección de $10 \mathrm{ml}$ de solución fisiológica y evaluación de: signos de aumento de resistencia a la inyección, aumento del perímetro de la extremidad, signos de extravasación, etc. En caso de producirse algún problema en el procedimiento debemos retirar la aguja e intentarlo en otra extremidad.

13.- Si el proceso ha sido exitoso, conexión al sistema de perfusión con alargadera y llave de tres vías, fijando el sistema a la piel.

14.- Retirar el acceso vascular intraoseo en cuanto logremos un acceso

intravenoso seguro
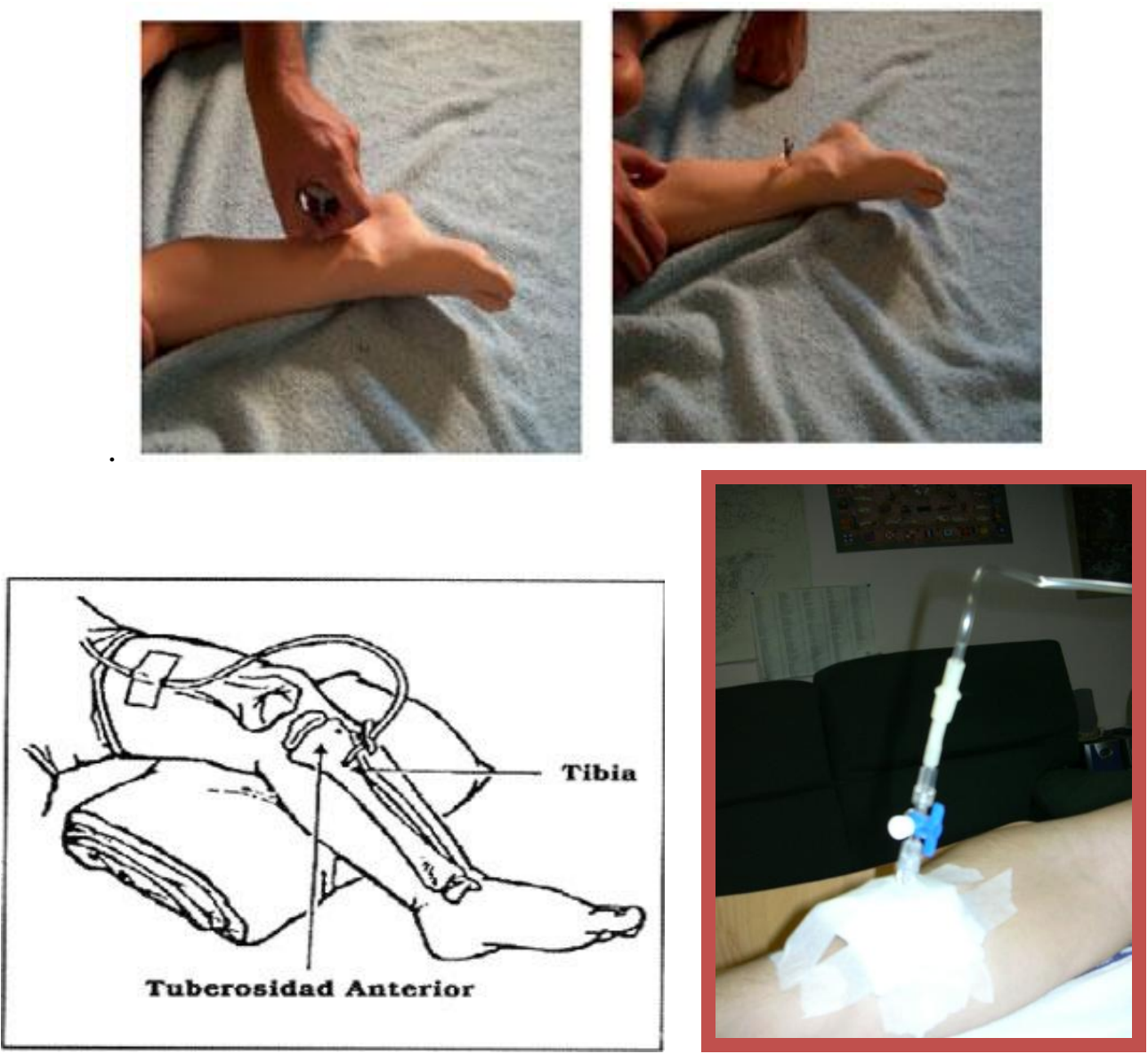


\section{REFERENCIAS BIBLIOGRÁFICAS}

1. American Academy of Pediatrics and American Heart Association. PALS: Provider manual. 2002

2. American Academy of Pediatrics and American Heart Association. PALS: Instructors manual. 2002

3. American Heart Association and American Academy of Pediatrics. Reanimación Avanzada Pediátrica. 1997-99

4. American Heart Association. Circulation 2000;102 (suppl I): I-1 - 241

5. Atkins D, Chameides L. Resuscitation sciencies of pediatrics. Ann Emerg Med. 2001;37:S41-S48

6. Brown K, Bocok J. Update in Pediatric resuscitation. Emerg Clin Nort Am 2002;20: 393-410

7. Rosetti V, Thompson BM, Arahaiman C. Difficulty and delay in intravascular access in pediatric arrest. Ann Emerg Med 1984;13:406.

8. Zimmerman JJ, Strauss RH. History and current application of intravenous therapy in children. Pediatr Emerg Care 1989;2:120.

9. Chameides L, Hazinzki M (ed). Reanimación Avanzada Pediátrica. Manual del Instructor Dallas, American Heart Association, 1998.

10. Rosetti V, Thompson BM, Arahaiman C. Difficulty and delay in intravascular access in pediatric arrest. Ann Emmerg Med 1984;13:406.

11. Rosetti V, Thompson BM, Miller J. Intraossoeus infusion: An alternative route of pediatric intravascular access. Ann Emerg Med 1985;14:885.

12. Recomendaciones de la National Association of EMS Physicians El acceso vascular intraóseo en un ámbito extrahospitalario. Prehospital Emergency Care (ed. esp.) 2008;1:101-3

13. Raymond Fowler, John V Gallagher, S Marshal Isaacs, Eric Ossman, Paul Pepe, Marvin Wayne. Valor del acceso vascular intraóseo en el contexto extrahospitalario. Prehospital Emergency Care (ed. esp.) 2008;1 : 95-100.

14. Alonso M, Aracil J. Punción intraósea. En: Benito J, Luaces C, Mintegi M, Pou $\mathrm{J}$ (eds). Tratado de urgencias pediátricas. Capítulo 4: Técnicas y procedimientos más habituales en urgencias de pediatría. 2004. 